\title{
STUDIES OF THE DIFFUSE INTERSTELLAR BANDS. IV. THE NEARLY PERFECT CORRELATION BETWEEN $\lambda \lambda 6196.0$ AND 6613.6
}

\author{
Benjamin J. McCall ${ }^{1}$, Meredith M. Drosback ${ }^{2}$, Julie A. Thorburn ${ }^{3}$, Donald G. York ${ }^{4}$, Scott D. Friedman $^{5}$, \\ L. M. Hobbs ${ }^{4}, 6$, Brian L. Rachford ${ }^{7}$, Theodore P. Snow ${ }^{2}$, Paule Sonnentrucker ${ }^{8}$, and Daniel E. Welty ${ }^{9}$

\begin{abstract}
In a sample of 114 diffuse cloud sightlines spanning a wide range of interstellar environments, we find the equivalent widths of the diffuse interstellar bands (DIBs) $\lambda 6196.0$ and $\lambda 6613.6$ to be extremely well correlated, with a correlation coefficient of 0.986 . A maximum likelihood functional relationship analysis shows that the observations are consistent with a perfect correlation if the observational errors, which are dominated by continuum placement and other systematics such as interfering lines, have been underestimated by a factor of 2 . The quality of this correlation far exceeds other previously studied correlations, such as that between the $\lambda 5780.5$ DIB and either the color excess or the atomic hydrogen column density. The unusually tight correlation between these two DIBs would seem to suggest that they might represent the first pair of DIBs known to be due to the same molecular carrier. However, further theoretical work will be required to determine whether the different linewidths and band shapes of these two DIBs can be consistent with a common carrier. If the two DIBs do not in fact share the same molecular carrier, their two carriers must be chemically very closely related.
\end{abstract}

Key words: ISM: lines and bands - ISM: molecules

\section{INTRODUCTION}

The diffuse interstellar bands (DIBs) were first discovered in the optical spectra of reddened stars in 1919 (Heger 1922), and remain the longest standing unsolved problem in spectroscopy. The scope of this problem is growing with time; recent surveys of the DIBs toward the heavily reddened stars HD 204827 (Hobbs et al. 2008, Paper II in this series) and HD 183143 (Hobbs et al. 2009, Paper III) have revealed a total of 380 and 414 DIBs in these sightlines, respectively.

Nearly 90 years of study have yielded numerous theories on possible carriers, including grain impurities and gas phase molecules or molecular ions, but no positive identification of any of the DIBs has been achieved (see reviews by Herbig 1995, Snow \& McCall 2006, and Sarre 2006 for more detailed discussions). Dedicated searches for individual molecular carriers have dominated recent astronomical and laboratory work on the problem, although there seems to be little doubt that the DIBs arise from many different molecular carriers.

Correlation studies on the DIBs have been performed for many years in an effort to extract some information about the physical nature of the DIB carriers. Numerous studies have compared individual DIBs to (1) known interstellar atomic and molecular species (see Herbig 1993; Thorburn et al. 2003, Paper II; Galazutdinov et al. 2004; and Weselek et al. 2008 for some examples), (2) interstellar line of sight properties, such as $E_{B-V}$ (dating as far back as Merrill \& Wilson 1938), and (3) other DIBs themselves (see Josafatsson \& Snow 1987 and Cami et al. 1997, for example).

Under the assumption that the DIBs have molecular carriers, the DIBs must represent vibronic transitions (with unresolved rotational fine structure) from a large number of different molecules. For a single molecule, the relative intensities of the vibronic bands that originate from the same vibrational energy level are governed only by their respective Franck-Condon factors (Herzberg 1950, pp. 194ff.). So, if we assume that all molecules giving rise to a particular DIB in a given sightline are in their ground vibrational state, then two (or more) DIBs should be part of a vibronic progression in the molecule, and the relative strengths of the vibronic bands should be precisely the same from one line of sight to another (assuming the bands are optically thin). We have been looking for correlations between the strengths of DIBs across a broad sample of sightlines in an effort to identify DIBs that could represent these vibronic bands within a single molecule. A tight correlation between a set of DIBs is a promising but insufficient condition for those DIBs to share a common molecular carrier - the width and shape of the (unresolved) rotational profiles of a given set of DIBs must also be consistent with the spectrum of a single molecule.

The assumption that all molecules of a single DIB carrier are in the ground vibrational state, however, may not be entirely valid in all instances. Under certain conditions, lowlying excited vibrational levels might become populated, and then the relative intensities of DIBs originating from the ground vibrational state and another low-lying excited vibrational state would depend not exclusively on Franck-Condon factors. The local conditions and relative populations of the states would then influence the intensities of the individual transitions, and their relative intensities would not be constant across all sightlines. Similarly, in a linear molecular carrier, a spin-orbit splitting of the ground electronic state could interfere with a correlation. However, those DIB carriers which do not have multiple 
low-lying populated energy levels can still be expected to show perfect correlations among their vibronic transitions.

This work is part of an extensive DIB survey conducted with the $3.5 \mathrm{~m}$ telescope at the Apache Point Observatory. Paper I in this series (Thorburn et al. 2003) identified a set of DIBs that are related to interstellar $\mathrm{C}_{2}$, and Papers II (Hobbs et al. 2008) and III (Hobbs et al. 2009) presented our first spectral atlases, toward the spectroscopic binary star HD 204827 and the well-studied star HD 183143. As part of this survey, we have begun a detailed study of the correlations between numerous DIBs, which will be presented in an upcoming publication. In the present Paper IV, we focus on the pair of DIBs with the highest correlation coefficient we have observed, namely the two DIBs we refer to as ${ }^{10} \lambda 6196.0$ and $\lambda 6613.6$, with adopted rest wavelengths of $6195.98 \AA$ and $6613.62 \AA$, according to Paper II (Hobbs et al. 2008).

In this paper, we revisit the correlation between the equivalent widths of these two DIBs now with a new, larger data set of 114 sightlines, most of which have not been used before to study this particular correlation, and with consistently high signal-tonoise ratio $(\mathrm{S} / \mathrm{N})$ spectra. Because of the modest resolution of our spectrometer, we will focus primarily on equivalent width measurements, rather than on an analysis of the band widths and shapes. The remainder of this paper will be organized as follows: Section 2 reviews the history of these two DIBs and their correlation, Section 3 presents the data and discusses the data reduction and the equivalent width measurements, Section 4 describes the statistical methods used to analyze the correlation, and Section 5 summarizes our results.

\section{THE $\lambda \lambda$ 6613.6 AND 6196.0 DIBS}

The DIB $\lambda 6613.6$ was among the first ever observed in interstellar spectra. An early published description of the DIBs seen in spectra taken at Mount Wilson included only the lines near 5780, 5797, 6284, and $6614 \AA$ (Merrill 1934). More refined observations over the years have been made in an effort not only to search for the presence of $\lambda 6613.6$ in individual sightlines, but also to measure its line profile. A study by Welter \& Savage (1977) was the first attempt to measure the profile of this DIB at high resolution $(\Delta \lambda \sim 0.2 \AA)$ in an effort to understand the physical process by which it may be produced. Welter $\&$ Savage were able to clearly discern the asymmetric structure of this DIB, but did not detect any of the detailed structure observed by later authors. Herbig \& Soderblom (1982) also noted the same asymmetry seen by Welter \& Savage, but with their higher resolution observations were the first to show that there appeared to be intrinsic structure within the band. Their spectra showed an "inflection" on the short wavelength side of the main absorption line, and they interpreted this as possible evidence of a weak component located approximately $0.3 \AA$ shortward of the dominant absorption.

More recent high resolution studies of the $\lambda 6613.6$ profile by Sarre et al. (1995), Ehrenfrend \& Foing (1996), Sonnentrucker et al. (1999), and Galazutdinov et al. (2002) have shown that

\footnotetext{
10 There has been persistent confusion in the literature regarding the nomenclature of the DIBs. Some authors truncate the rest wavelengths to the nearest $\AA$ (e.g., $6613.62 \rightarrow \lambda 6613$ ), while others round them (e.g., 6613.62 $\rightarrow \lambda 6614)$. Since these unidentified bands have unknown rest wavelengths and band structures, it is difficult to adopt a uniform convention; the case of $\lambda 6196.0$ is especially problematic, as different measurements of the central wavelength have ranged from 6195.95 (Herbig 1975) to 6196.19 (Jenniskens $\&$ Désert 1994). We have decided to adopt a new convention, using five significant figures, and rounding the rest wavelengths from Paper II (Hobbs et al. 2008).
}

the DIB is composed of three individual components, each separated by roughly $0.3 \AA$, and with an overall FWHM of $\sim 0.9 \AA$. This structure has been interpreted by both Sarre et al. and Galazutdinov et al. as evidence of rotational branch structure in a gas-phase molecule. Molecular rotational contour fitting was performed by Kerr et al. (1996) on the profile of the DIB in order to investigate the physical properties, such as molecular constants and rotational temperatures, of a molecule consistent with the observed profile. An alternative explanation for the internal structure was presented by Webster (1996); he demonstrated that the observed structure could be created by an isotopic mix of ${ }^{12} \mathrm{C}$ and ${ }^{13} \mathrm{C}$ in a large carbon-based molecule. Later investigations by Walker et al. (2000) presented more high resolution profiles of $\lambda 6613.6$ and successfully fit the profile with multiple Gaussians of equal spacing, equal width and a Poissonian intensity distribution, as predicted in the Webster isotope model. However, subsequent studies by Cami et al. (2004) found variations in the spacing between the three components, which is inconsistent with the isotope model.

The first mention of $\lambda 6196.0$ appeared in the 1975 DIB survey by Herbig. Called the "sharpest of the diffuse lines" then known, it also appeared to show no signs of asymmetry, unlike $\lambda 6613.6$ (Herbig 1975). The $\lambda 6196.0$ profile was studied at high resolution $(\sim 70,000)$ by Smith et al. (1981) in twenty six reddened sightlines to search for any evidence of fine structure within the band; no fine structure was found, but a nearby weak absorption feature was discovered and later attributed to another DIB. Smith et al. note that while the central wavelength of $\lambda 6196.0$ appears to be stable, the width is not constant, and instead appears generally to widen with increasing interstellar reddening (though HD 187982 is a notable exception to this). A separate study by Herbig \& Soderblom (1982) of the profile of $\lambda 6196.0$ confirmed the results of Smith et al., finding that $\lambda 6196.0$ has a narrow (full width at half-maximum $\sim 0.35 \AA$ ), symmetric profile. In the same study with the $\lambda 6613.6$ profile work, Galazutdinov et al. (2002) presented high resolution observations of the $\lambda 6196.0$ profile that suggest there is some evidence of substructure within this DIB as well, though it is not as well-defined as that observed for the broader DIB $\lambda 6613.6$.

Like the rest of the DIBs, $\lambda 6196.0$ and $\lambda 6613.6$ are generally considered to be caused by interstellar molecules. While there has been no dispute about their interstellar origin, the observation in Paper II (Hobbs et al. 2008) that they are stationary in the line of sight towards HD 204827 has removed any doubt. The compelling argument in favor of a gas-phase molecular origin for $\lambda 6196.0$ is the stability of the measured wavelength across numerous sightlines (Smith et al. 1981). If a DIB were due to a solid-state process, one would expect to observe shifts in the wavelength of the feature across many sightlines as a result of physical differences in the host grains in different clouds (Smith et al. 1977). The same wavelength stability is generally observed for $\lambda 6613.6$. In addition, the intrinsic structure in this band provides strong support for the molecular hypothesis; both suggested explanations for this structure rely on the assumption of a molecular carrier. Intriguingly, both bands have been shown to exhibit profile variations (Galazutdinov et al. 2008b, 2008c), as well as blue shifts in the Sco OB1 association (Galazutdinov et al. 2008a).

These two DIBs have already been shown to have a particularly tight correlation in their equivalent widths. Moutou et al. (1999) first noticed the strong correlation in a larger search for correlations between the DIBs. The authors included 62 sightlines in their work, 43 of which overlap with those presented 
in this paper. Montou et al. found a correlation coefficient of 0.97 when using measured central depths, and 0.98 using equivalent widths. They concluded that the tight correlation is a real effect and it implies these two DIBs result from either the same molecule or carriers that are closely linked. Later work by Galazutdinov et al. (2002) followed up on the correlation observed by Moutou et al. and studied the profiles of these two DIBs at high resolution. They determined, using observations of 7 sightlines, that the profiles of the two DIBs appear to be changed by different mechanisms and postulate that, as a result, $\lambda 6196.0$ and $\lambda 6613.6$ are unlikely to be due to the same molecule.

\section{OBSERVATIONS AND DATA REDUCTION}

The data used in this study were obtained using the Astrophysical Research Consortium Echelle Spectrograph (ARCES) at the $3.5 \mathrm{~m}$ telescope at Apache Point Observatory between 1999 and 2003. The ARCES spectrograph has a resolving power of 38,000 (corresponding to a velocity resolution of approximately $8 \mathrm{~km} \mathrm{~s}^{-1}$ ) and in a single exposure covers the spectral range from $3500 \AA$ to $10000 \AA$ (Wang et al. 2003). The spectra were taken as part of a larger initiative to amass a database of high quality spectra of the DIBs. The goal of this long term DIB survey was to achieve an $\mathrm{S} / \mathrm{N}$ of 1000 at $5780 \AA$ in each sightline. A minimum of four exposures was required for each sightline to reach this $\mathrm{S} / \mathrm{N}$ level, and the individual images were combined during the data reduction process. The data reduction on all of the spectra in the DIB database was done with NOAO's Image Reduction and Analysis Facility (IRAF) ${ }^{11}$. The rest frame for the composite spectrum of each sightline was set by shifting the strongest component of the K I line (7698.97 $\AA$ ) in each individual spectrum to its rest wavelength before coaddition. In cases where multiple $\mathrm{K}$ I components are present, a DIB may appear to have different rest wavelengths if the strongest $\mathrm{K}_{\mathrm{I}}$ component does not contain the most carriers of that DIB. A detailed discussion of the data reduction process can be found in Paper I (Thorburn et al. 2003).

A total of 114 sightlines in the compiled DIB database contained detections of both $\lambda 6196.0$ and $\lambda 6613.6$. Table 1 lists these sightlines and several pertinent physical parameters, such as spectral type, color excess, and atomic and molecular hydrogen column densities for each sightline. The sightlines in the larger DIB survey were chosen to sample a wide range of physical interstellar parameters, including molecular hydrogen fraction $\left(f_{\mathrm{H}_{2}}^{N}\right)$, interstellar reddening, and interstellar extinction. The 114 sightlines included in the present study also represent a diverse sample of the interstellar medium in order to study the correlation of these two DIBs across a broad range of interstellar conditions. The fraction of molecular hydrogen in our sample ranges from a low of $2.6 \times 10^{-6}$ (a sightline dominated by atomic hydrogen) to a high of 0.76 (a sightline with mostly molecular hydrogen); the color excess, $E_{B-V}$ covers a range of 0.02-3.31. Of the 114 sightlines, the majority $(68 \%)$ are toward B stars. The remaining sightlines are toward O stars (29\%) and A stars (3\%). Our sample contains sightlines with only one $\mathrm{K}_{\mathrm{I}}$ component, as well as sightlines with multiple (up to 17) $\mathrm{K}_{\mathrm{I}}$ components (D. E. Welty, private communication).

Figures 1 and 2 show six representative examples of each DIB in order to illustrate some of the observed variations in the

\footnotetext{
11 IRAF is distributed by the NOAO, which is operated by the Association of Universities for Research in Astronomy, Inc., under cooperative agreement with the National Science Foundation.
}

surrounding spectrum. The two DIBs discussed in this study, $\lambda 6196.0$ and $\lambda 6613.6$, are labeled, as are the other DIBs located in the plotted range. The minor DIB at $6194.9 \AA$ is not included in this study, although it will be discussed below in terms of its impact on the measurement of the equivalent width of $\lambda 6196.0$. Also plotted in each panel, and offset vertically for ease of comparison, are representative lightly reddened sightlines of similar spectral type to the DIB sightlines. We present these lowreddening sightlines in order to illustrate what the underlying stellar spectrum looks like in the vicinity of $\lambda 6196.0$ and $\lambda$ 6613.6. There are no obviously strong stellar features in the regions around these two DIBs in any of our sample of stars.

In order to discuss the correlation between these two DIBs with any level of confidence, we had to measure the equivalent widths as accurately as possible. In both cases, there are nearby lines whose interference had to be taken into account when measuring the equivalent widths of the DIBs. In the $\lambda 6196.0$ case in particular, there is another DIB centered near $6194.9 \AA{ }^{12}$ There are no interfering telluric lines in the spectral regions surrounding either $\lambda 6196.0$ or $\lambda 6613.6$, therefore no special telluric correction had to be performed for this analysis. The resolution of ARCES is not sufficient to resolve the level of detailed structure in $\lambda 6613.6$ that other studies have shown, but the asymmetry is obviously preserved. In a few cases, we do see a hint of the substructure of this DIB, as shown in Figure 3. ARCES's resolution is insufficient to observe any of the structure in the $\lambda 6196.0$ profile as observed by Galazutdinov et al. (2002).

To measure the equivalent width of the DIBs, first the surrounding continuum was fitted and normalized. Then, in cases where the wings of the nearby lines appeared to overlap with the DIB of interest, these interfering lines were fitted with a Gaussian and subtracted out of the spectrum. The equivalent widths of the DIBs were measured by a straightforward integration across the profile of the DIB, with limits of integration that varied from one sightline to another but were consistently set by one of us (M.M.D.) in all sightlines in one sitting. Table 1 includes the measured equivalent widths of $\lambda 6196.0$ and $\lambda 6613.6$ for each of the sightlines in this study. We note that the equivalent width measurements reported in this analysis are independent of those presented in Paper I (Thorburn et al. 2003). The latter measurements used the same fixed integration limits for all sightlines, but the two sets generally agree within their mutual uncertainties, with correlation coefficients of 0.996 between the two sets of $\lambda 6196.0$ measurements, and 0.995 between the $\lambda 6613.6$ measurements.

The estimation of the errors in the equivalent width measurements is a challenge, because the true continuum level is not known a priori, because the true bandshape (and therefore appropriate limits of integration) of the DIBs is unknown, and because the DIBs we are measuring are likely to be contaminated by weaker and possibly unresolved interfering lines, which could be stellar or interstellar. These systematic errors are difficult to quantify, and likely dominate the statistical error that is due to integration across the line profile, which can be assessed using the random rms noise as measured in the surrounding continuum outside the region of integration. As a crude attempt to quantify the systematic errors, we have taken the rms uncertainty in the continuum fit and determined the additional

\footnotetext{
12 The effect of this overlapping DIB can be seen most clearly in the case of HD 223385 in Figure 1. This particular sightline is the worst outlier above the regression line in Figure 5, perhaps because we have underestimated $W_{\lambda}(6196.0)$ by overestimating the contribution of this interfering DIB.
} 
Table 1

Physical Properties and Equivalent Width Measurements for Program Stars

\begin{tabular}{|c|c|c|c|c|c|c|c|}
\hline Star & Spectral Type & $E_{B-V}{ }^{\mathrm{a}}$ & $N(\mathrm{H})$ & $N\left(\mathrm{H}_{2}\right)$ & References $^{\mathrm{b}}$ & $W_{\lambda}(6196.0)(\mathrm{m} \AA)$ & $W_{\lambda}(6613.6)(\mathrm{m} \AA)$ \\
\hline HD 2905 & B1Iae & 0.33 & $21.26 \pm 0.09$ & $20.27 \pm 0.09$ & 3,6 & $30.9 \pm 1.6$ & $131.1 \pm 2.9$ \\
\hline HD 19374 & B $1.5 \mathrm{~V}$ & 0.13 & $21.06 \pm 0.11$ & $\ldots$ & $1,-$ & $13.8 \pm 1.3$ & $37.0 \pm 2.6$ \\
\hline HD 20041 & A0Ia & 0.72 & $\ldots$ & $\ldots$ & $\ldots$ & $56.7 \pm 1.7$ & $253.8 \pm 4.5$ \\
\hline HD 21071 & B7V & 0.05 & $\ldots$ & $\ldots$ & $\ldots$ & $9.1 \pm 1.2$ & $28.2 \pm 3.1$ \\
\hline HD 21483 & B3III & 0.56 & $\ldots$ & $\ldots$ & $\ldots$ & $23.2 \pm 1.6$ & $90.7 \pm 4.3$ \\
\hline HD 21389 & A0Iae & 0.57 & $\ldots$ & $\ldots$ & $\ldots$ & $43.8 \pm 2.1$ & $168.6 \pm 5.1$ \\
\hline HD 22951 & B $0.5 \mathrm{~V}$ & 0.27 & $21.04 \pm 0.11$ & $20.46 \pm 0.09$ & 5,6 & $18.7 \pm 1.8$ & $52.4 \pm 2.8$ \\
\hline HD 23180 & B1III & 0.31 & $20.85 \pm 0.09$ & $20.61 \pm 0.09$ & 1,6 & $15.1 \pm 1.2$ & $56.6 \pm 2.7$ \\
\hline HD 281159 & $\mathrm{~B} 5 \mathrm{~V}$ & 0.85 & $21.38 \pm 0.3$ & $21.09 \pm 0.19$ & 9,9 & $34.0 \pm 1.2$ & $159.2 \pm 2.5$ \\
\hline HD 23408 & B8III & 0.02 & $\ldots$ & $19.75 \pm 0.13$ &,- 6 & $1.9 \pm 0.5$ & $17.1 \pm 3.1$ \\
\hline HD 24398 & $\mathrm{~B} 1 \mathrm{Ib}$ & 0.31 & $20.8 \pm 0.08$ & $20.68 \pm 0.09$ & 1,6 & $17.4 \pm 1.8$ & $61.6 \pm 3.3$ \\
\hline HD 24534 & O9.5pe & 0.59 & $20.73 \pm 0.06$ & $20.92 \pm 0.04$ & 1,8 & $15.9 \pm 1.3$ & $72.5 \pm 4.4$ \\
\hline HD 24760 & $\mathrm{~B} 0.5 \mathrm{~V}+\mathrm{A} 2$ & 0.1 & $20.45 \pm 0.11$ & $19.52 \pm 0.13$ & 1,6 & $8.0 \pm 1.8$ & $17.1 \pm 2.5$ \\
\hline HD 24912 & O7e & 0.33 & $21.05 \pm 0.08$ & $20.54 \pm 0.08$ & 1,6 & $21.3 \pm 1.2$ & $81.0 \pm 3.1$ \\
\hline HD 26571 & B9IIIp & 0.25 & $\ldots$ & $\ldots$ & $\ldots$ & $20.6 \pm 1.2$ & $84.6 \pm 3.7$ \\
\hline HD 28375 & B3V & 0.1 & $\ldots$ & $\ldots$ & $\ldots$ & $6.6 \pm 1.1$ & $17.7 \pm 3.1$ \\
\hline HD 29647 & B8IIIp & 1 & $\ldots$ & $\ldots$ & $\ldots$ & $12.1 \pm 1.6$ & $62.3 \pm 4.4$ \\
\hline HD 30614 & O9.5Iae & 0.3 & $20.97 \pm 0.09$ & $20.34 \pm 0.08$ & 1,6 & $16.1 \pm 1.0$ & $74.9 \pm 3.4$ \\
\hline HD 34078 & O9.5Ve & 0.52 & $21.2 \pm 0.11$ & $\ldots$ & $1,-$ & $25.4 \pm 2.2$ & $63.8 \pm 3.3$ \\
\hline HD 35149 & $\mathrm{~B} 1 \mathrm{~V}$ & 0.11 & $20.56 \pm 0.07$ & $18.3 \pm 0.11$ & 1,9 & $6.2 \pm 0.7$ & $19.6 \pm 1.9$ \\
\hline HD 36591 & B1IV & 0.07 & $\ldots$ & $\ldots$ & $\ldots$ & $10.1 \pm 1.9$ & $10.8 \pm 2.7$ \\
\hline HD 36371 & B5Iab & 0.43 & $\ldots$ & $\ldots$ & $\ldots$ & $39.2 \pm 1.9$ & $146.8 \pm 3.8$ \\
\hline HD 36822 & B0III & 0.14 & $20.84 \pm 0.07$ & $19.32 \pm 0.07$ & 1,6 & $8.8 \pm 1.0$ & $23.5 \pm 2.2$ \\
\hline HD 36861 & O8e & 0.15 & $20.81 \pm 0.12$ & $19.12 \pm 0.1$ & 1,6 & $5.6 \pm 1.3$ & $19.5 \pm 3.1$ \\
\hline HD 37022 & O6 & 0.34 & $21.54 \pm 0.11$ & $15.65 \pm 0.13$ & 1,7 & $6.4 \pm 1.0$ & $16.2 \pm 3.0$ \\
\hline HD 37043 & O9III & 0.07 & $20.2 \pm 0.1$ & $14.69 \pm 0.11$ & 1,7 & $3.8 \pm 1.0$ & $4.8 \pm 1.9$ \\
\hline HD 37061 & $\mathrm{~B} 1 \mathrm{~V}$ & 0.52 & $21.82 \pm 0.07$ & $\ldots$ & $3,-$ & $12.6 \pm 1.5$ & $35.2 \pm 5.9$ \\
\hline HD 37128 & B0Iae & 0.05 & $20.48 \pm 0.11$ & $16.28 \pm 0.2$ & 1,9 & $3.2 \pm 0.6$ & $10.1 \pm 2.3$ \\
\hline HD 37367 & B2IV-V & 0.4 & $\ldots$ & $\ldots$ & $\ldots$ & $41.5 \pm 1.9$ & $149.8 \pm 4.6$ \\
\hline HD 37742 & O9.5Ibe & 0.06 & $20.39 \pm 0.09$ & $15.88 \pm 0.11$ & 1,9 & $3.2 \pm 0.7$ & $6.8 \pm 2.8$ \\
\hline HD 38771 & B0.5Ia & 0.05 & $20.6 \pm 0.08$ & $15.68 \pm 0.14$ & 1,7 & $4.6 \pm 0.8$ & $9.6 \pm 1.7$ \\
\hline HD 40111 & B0.5II & 0.2 & $21.03 \pm 0.09$ & $19.73 \pm 0.1$ & 1,6 & $15.7 \pm 5.3$ & $39.4 \pm 10.2$ \\
\hline HD 41117 & B2Iae & 0.45 & $21.4 \pm 0.15$ & $20.69 \pm 0.1$ & 1,10 & $41.3 \pm 0.9$ & $158.8 \pm 4.2$ \\
\hline HD 42087 & B2.5Ibe & 0.36 & $21.4 \pm 0.11$ & $20.52 \pm 0.12$ & 1,10 & $31.2 \pm 1.5$ & $121.9 \pm 3.0$ \\
\hline HD 43247 & B9II-III & 0.03 & $\ldots$ & $\ldots$ & $\ldots$ & $9.9 \pm 1.3$ & $26.3 \pm 3.5$ \\
\hline HD 43384 & $\mathrm{~B} 3 \mathrm{Ib}$ & 0.58 & $\ldots$ & $20.87 \pm 0.14$ &,- 10 & $48.1 \pm 1.6$ & $201.5 \pm 4.2$ \\
\hline HD 46056 & O8V & 0.5 & $21.38 \pm 0.14$ & $20.68 \pm 0.06$ & 1,10 & $34.7 \pm 2.1$ & $141.0 \pm 4.7$ \\
\hline HD 46202 & $\mathrm{O} 9 \mathrm{~V}$ & 0.49 & $21.58 \pm 0.15$ & $20.68 \pm 0.06$ & 1,10 & $36.5 \pm 2.0$ & $135.7 \pm 3.8$ \\
\hline HD 46711 & B3II & 1.04 & $\ldots$ & $\ldots$ & $\ldots$ & $85.7 \pm 4.6$ & $359.4 \pm 6.6$ \\
\hline HD 47129 & $\mathrm{O} 8 \mathrm{~V}+\mathrm{O} 8 \mathrm{f}$ & 0.36 & $21.18 \pm 0.11$ & $20.55 \pm 0.09$ & 1,6 & $22.8 \pm 1.3$ & $100.6 \pm 4.6$ \\
\hline HD 48099 & O6e & 0.27 & $21.2 \pm 0.12$ & $20.29 \pm 0.07$ & 1,7 & $20.6 \pm 1.4$ & $78.8 \pm 3.0$ \\
\hline HD 50064 & B6Ia & 0.85 & $\ldots$ & $\ldots$ & $\ldots$ & $71.3 \pm 6.0$ & $279.4 \pm 6.9$ \\
\hline HD 51309 & B3II & 0.11 & $\ldots$ & $\ldots$ & $\ldots$ & $8.0 \pm 1.5$ & $15.9 \pm 4.2$ \\
\hline HD 53367 & B0.5III & 0.52 & $\ldots$ & $21.04 \pm 0.05$ &,- 10 & $24.2 \pm 1.3$ & $83.6 \pm 2.1$ \\
\hline HD 53975 & $\mathrm{O} 8 \mathrm{~V}$ & 0.21 & $21.1 \pm 0.08$ & $19.23 \pm 0.09$ & 1,6 & $17.2 \pm 1.3$ & $51.5 \pm 3.2$ \\
\hline HD 54662 & O7III & 0.35 & $21.23 \pm 0.1$ & $20 \pm 0.09$ & 1,6 & $25.5 \pm 1.9$ & $94.5 \pm 3.5$ \\
\hline HD 57060 & $\mathrm{O} 7 \mathrm{e}+\mathrm{O} 7$ & 0.17 & $20.78 \pm 0.1$ & $15.78 \pm 0.1$ & 1,7 & $7.1 \pm 1.2$ & $11.4 \pm 1.9$ \\
\hline HD 57061 & O9III & 0.16 & $20.8 \pm 0.08$ & $15.45 \pm 0.13$ & 1,7 & $6.7 \pm 1.1$ & $12.1 \pm 2.5$ \\
\hline HD 91316 & $\mathrm{~B} 1 \mathrm{Ib}$ & 0.05 & $20.44 \pm 0.09$ & $15.58 \pm 0.08$ & 1,6 & $4.8 \pm 1.3$ & $10.0 \pm 1.7$ \\
\hline HD 143275 & B0.3IV & 0.17 & $21.01 \pm 0.08$ & $19.42 \pm 0.1$ & 1,6 & $8.9 \pm 1.6$ & $23.4 \pm 3.2$ \\
\hline HD 144217 & $\mathrm{~B} 1 \mathrm{~V}$ & 0.19 & $21.03 \pm 0.08$ & $19.83 \pm 0.04$ & 1,6 & $14.6 \pm 1.6$ & $40.9 \pm 3.0$ \\
\hline HD 144218 & $\mathrm{~B} 2 \mathrm{~V}$ & 0.22 & $\ldots$ & $\ldots$ & $\ldots$ & $15.3 \pm 1.6$ & $53.1 \pm 4.4$ \\
\hline HD 144470 & $\mathrm{~B} 1 \mathrm{~V}$ & 0.22 & $21.18 \pm 0.08$ & $20.06 \pm 0.06$ & 5,6 & $17.7 \pm 1.6$ & $63.0 \pm 3.0$ \\
\hline HD 145502 & B3V & 0.24 & $21.2 \pm 0.12$ & $19.89 \pm 0.07$ & 1,6 & $17.0 \pm 1.3$ & $69.4 \pm 3.5$ \\
\hline HD 147165 & $\mathrm{~B} 2 \mathrm{III}+\mathrm{O} 9 \mathrm{~V}$ & 0.41 & $21.38 \pm 0.08$ & $19.79 \pm 0.07$ & 1,6 & $21.6 \pm 1.7$ & $67.7 \pm 4.3$ \\
\hline HD 147888 & B5V & 0.47 & $\ldots$ & $20.47 \pm 0.05$ &,- 10 & $20.2 \pm 1.1$ & $78.5 \pm 2.2$ \\
\hline HD 147889 & $\mathrm{~B} 2 \mathrm{~V}$ & 1.07 & $\ldots$ & $\ldots$ & $\ldots$ & $45.5 \pm 1.7$ & $189.0 \pm 4.5$ \\
\hline HD 147933 & B2IV & 0.48 & $21.63 \pm 0.09$ & $20.57 \pm 0.07$ & 1,6 & $17.1 \pm 1.6$ & $70.7 \pm 4.2$ \\
\hline HD 148184 & B2IVpe & 0.52 & $<21.23$ & $20.63 \pm 0.09$ & 2,6 & $14.5 \pm 1.6$ & $50.4 \pm 6.3$ \\
\hline HD 149404 & O9Iae & 0.68 & $21.4 \pm 0.14$ & $20.79 \pm 0.04$ & 1,10 & $45.2 \pm 2.4$ & $163.7 \pm 4.8$ \\
\hline
\end{tabular}


Table 1

(Continued)

\begin{tabular}{|c|c|c|c|c|c|c|c|}
\hline Star & Spectral Type & $E_{B-V}^{\mathrm{a}}$ & $N(\mathrm{H})$ & $N\left(\mathrm{H}_{2}\right)$ & References $^{\mathrm{b}}$ & $\bar{W}_{\lambda}(6196.0)(\mathrm{m \AA})$ & $W_{\lambda}(6613.6)(\mathrm{m} \AA)$ \\
\hline HD 159975 & B8II-IIIp & 0.19 & $\ldots$ & $\ldots$ & $\ldots$ & $18.2 \pm 1.2$ & $71.4 \pm 3.9$ \\
\hline HD 162978 & O8III & 0.35 & $21.28 \pm 0.08$ & $\ldots$ & $1,-$ & $18.6 \pm 1.7$ & $74.3 \pm 7.2$ \\
\hline HD 164353 & $\mathrm{~B} 5 \mathrm{Ib}$ & 0.11 & $<21$ & $20.26 \pm 0.14$ & 5,6 & $14.3 \pm 0.8$ & $52.5 \pm 2.1$ \\
\hline HD 164740 & $07.5 \mathrm{~V}$ & 0.87 & $\ldots$ & $20.19 \pm 0.12$ &,- 10 & $30.7 \pm 3.0$ & $143.8 \pm 15.2$ \\
\hline HD 166734 & O8e & 1.39 & $\ldots$ & $\ldots$ & $\ldots$ & $95.2 \pm 1.7$ & $397.1 \pm 4.2$ \\
\hline HD 166937 & B8Iape & 0.25 & $\ldots$ & $\ldots$ & $\ldots$ & $25.9 \pm 1.2$ & $89.5 \pm 3.9$ \\
\hline HD 167971 & O8e & 1.08 & $21.6 \pm 0.3$ & $20.85 \pm 0.12$ & 8,8 & $55.9 \pm 1.1$ & $220.7 \pm 6.0$ \\
\hline HD 168076 & O5f & 0.78 & $21.65 \pm 0.23$ & $20.68 \pm 0.08$ & 1,8 & $61.4 \pm 1.7$ & $234.5 \pm 8.1$ \\
\hline HD 169454 & B 1.5Ia & 1.12 & $\ldots$ & $\ldots$ & $\ldots$ & $56.0 \pm 2.6$ & $216.0 \pm 5.2$ \\
\hline HD 170740 & $\mathrm{~B} 2 \mathrm{~V}$ & 0.48 & $21.04 \pm 0.15$ & $20.86 \pm 0.08$ & 1,8 & $28.4 \pm 1.4$ & $126.5 \pm 4.0$ \\
\hline HD 172028 & $\mathrm{~B} 2 \mathrm{~V}$ & 0.79 & $\ldots$ & $\ldots$ & $\ldots$ & $41.3 \pm 1.9$ & $145.8 \pm 6.5$ \\
\hline HD 175156 & B5II & 0.31 & $\ldots$ & $\ldots$ & $\ldots$ & $19.2 \pm 0.8$ & $68.9 \pm 2.9$ \\
\hline HD 179406 & B3V & 0.33 & $\ldots$ & $20.73 \pm 0.07$ &,- 10 & $21.5 \pm 1.2$ & $104.9 \pm 5.0$ \\
\hline HD 183143 & B7Iae & 1.27 & $\ldots$ & $\ldots$ & $\ldots$ & $90.2 \pm 1.2$ & $338.2 \pm 3.6$ \\
\hline HD 192639 & O8e & 0.66 & $21.32 \pm 0.12$ & $20.69 \pm 0.05$ & 1,8 & $38.1 \pm 1.0$ & $151.7 \pm 7.1$ \\
\hline HD 229059 & B1.5Iap & 1.71 & $\ldots$ & $\ldots$ & $\ldots$ & $65.0 \pm 1.4$ & $245.3 \pm 7.1$ \\
\hline HD 194839 & B0.5Ia & 1.18 & $\ldots$ & $\ldots$ & $\ldots$ & $57.4 \pm 3.0$ & $170.4 \pm 7.0$ \\
\hline Cyg OB2 5 & O7f & 1.99 & $\ldots$ & $\ldots$ & $\ldots$ & $88.5 \pm 2.4$ & $301.5 \pm 10.9$ \\
\hline Cyg OB2 12 & B5Ie & 3.31 & $\ldots$ & $\ldots$ & $\ldots$ & $106.4 \pm 9.8$ & $389.4 \pm 17.3$ \\
\hline HD 198478 & B3Iae & 0.54 & $\ldots$ & $\ldots$ & $\ldots$ & $34.9 \pm 1.4$ & $146.0 \pm 5.0$ \\
\hline HD 199579 & O6Ve & 0.37 & $21.04 \pm 0.11$ & $20.53 \pm 0.04$ & 1,8 & $15.8 \pm 1.0$ & $59.5 \pm 3.7$ \\
\hline HD 201345 & $09.5 \mathrm{~V}$ & 0.18 & $\ldots$ & $\ldots$ & $\ldots$ & $10.7 \pm 1.8$ & $24.3 \pm 4.7$ \\
\hline HD 202850 & B9Iab & 0.12 & $\ldots$ & $\ldots$ & $\ldots$ & $15.7 \pm 1.1$ & $48.9 \pm 4.1$ \\
\hline HD 203938 & B0.5IV & 0.74 & $21.48 \pm 0.15$ & $21 \pm 0.06$ & 8,8 & $43.3 \pm 1.1$ & $147.3 \pm 6.0$ \\
\hline HD 204172 & B0Ib & 0.16 & $21 \pm 0.11$ & $19.6 \pm 0.09$ & 5,6 & $10.9 \pm 0.8$ & $37.6 \pm 3.7$ \\
\hline HD 204827 & B0V & 1.11 & $\ldots$ & $\ldots$ & $\ldots$ & $41.9 \pm 1.5$ & $174.7 \pm 5.4$ \\
\hline HD 206165 & $\mathrm{~B} 2 \mathrm{Ib}$ & 0.47 & $\ldots$ & $\ldots$ & $\ldots$ & $27.4 \pm 1.6$ & $119.5 \pm 3.9$ \\
\hline HD 206267 & O6f & 0.53 & $21.3 \pm 0.15$ & $20.86 \pm 0.04$ & 8,8 & $30.2 \pm 1.1$ & $120.9 \pm 4.3$ \\
\hline HD 208440 & $\mathrm{~B} 1 \mathrm{~V}$ & 0.33 & $\ldots$ & $\ldots$ & $\ldots$ & $23.8 \pm 2.1$ & $108.2 \pm 7.1$ \\
\hline HD 208501 & $\mathrm{~B} 8 \mathrm{Ib}$ & 0.75 & $\ldots$ & $\ldots$ & $\ldots$ & $38.8 \pm 2.5$ & $131.9 \pm 7.8$ \\
\hline HD 209008 & B3III & 0.08 & $\ldots$ & $\ldots$ & $\ldots$ & $4.9 \pm 1.0$ & $13.0 \pm 2.6$ \\
\hline HD 209975 & O9Ib & 0.36 & $21.17 \pm 0.09$ & $20.08 \pm 0.09$ & 1,6 & $28.7 \pm 2.2$ & $120.0 \pm 8.6$ \\
\hline HD 210121 & B3V & 0.4 & $20.63 \pm 0.15$ & $20.75 \pm 0.12$ & 8,8 & $11.2 \pm 1.3$ & $28.4 \pm 2.2$ \\
\hline HD 210839 & O6If & 0.57 & $21.15 \pm 0.12$ & $20.84 \pm 0.04$ & 1,8 & $32.3 \pm 1.5$ & $158.5 \pm 7.1$ \\
\hline HD 212120 & B6V & 0.04 & $\ldots$ & $\ldots$ & $\ldots$ & $4.0 \pm 0.9$ & $12.1 \pm 2.4$ \\
\hline HD 212791 & B3V & 0.17 & $\ldots$ & $\ldots$ & $\ldots$ & $12.7 \pm 1.4$ & $43.6 \pm 5.2$ \\
\hline HD 214680 & $\mathrm{O} 9 \mathrm{~V}$ & 0.11 & $20.69 \pm 0.14$ & $19.22 \pm 0.06$ & 1,6 & $7.7 \pm 1.4$ & $14.2 \pm 2.9$ \\
\hline HD 214930 & B2IV & 0.1 & $\ldots$ & $\ldots$ & $\ldots$ & $7.9 \pm 1.7$ & $17.9 \pm 4.4$ \\
\hline HD 215733 & B1II & 0.11 & $20.75 \pm 0.09$ & $19.45 \pm 0.1$ & 1,9 & $10.1 \pm 1.5$ & $19.6 \pm 3.1$ \\
\hline HD 218376 & B0.5IV & 0.25 & $20.91 \pm 0.09$ & $20.15 \pm 0.09$ & 1,6 & $15.9 \pm 1.0$ & $70.0 \pm 2.5$ \\
\hline HD 219188 & B0.5II & 0.13 & $20.75 \pm 0.09$ & $19.38 \pm 0.12$ & 1,6 & $7.1 \pm 0.6$ & $21.8 \pm 2.5$ \\
\hline BD+63 1964 & BOII & 1 & $\ldots$ & $\ldots$ & $\ldots$ & $89.6 \pm 2.2$ & $334.0 \pm 5.8$ \\
\hline HD 223385 & A3Iae & 0.67 & $\ldots$ & $\ldots$ & $\ldots$ & $40.2 \pm 0.9$ & $201.9 \pm 5.2$ \\
\hline HD 224572 & $\mathrm{~B} 1 \mathrm{~V}$ & 0.19 & $20.79 \pm 0.08$ & $20.23 \pm 0.09$ & 1,6 & $10.3 \pm 0.9$ & $29.6 \pm 25$ \\
\hline
\end{tabular}

Notes.

a The calculated $E_{B-V}$ values are based on the intrinsic colors from Johnson (1963).

b The references are given for $N(\mathrm{H})$ and $N\left(\mathrm{H}_{2}\right)$, respectively.

References. (1) Diplas \& Savage 1994, Table 1; (2) Diplas \& Savage 1994, Table 2; (3) Shull \& van Steenberg 1985, Table 1; (4) Shull \& van Steenberg 1985, Table 2; (5) Bohlin et al. 1978; (6) Savage, et al. 1977; (7) Spitzer et al. 1974; (8) Rachford et al. 2002; (9) B. L. Rachford, private communication; (10) Rachford et al. 2009.

equivalent width that would result from a continuum displacement of that magnitude; this is essentially the "rms shift" method described by Sembach \& Savage (1992). In all sightlines, this estimate of the systematic error dominated the statistical error by approximately a factor of 10 . This error estimate is illustrated graphically in Figure 4, where the effect of displacing the continuum by twice (for clarity) our estimated error is shown. In the following analyses, we first assume that this estimated sys- tematic error constitutes the total uncertainty in the equivalent width measurements, and then we consider the possibility that the total uncertainty might be twice this value. Nevertheless, we caution the reader that these could easily be underestimates of the true errors, considering the potential effects of blending by other weak DIBs or other weak lines in the "continuum." A more rigorous and complete treatment of various potential systematic error sources will be pursued in future work. 


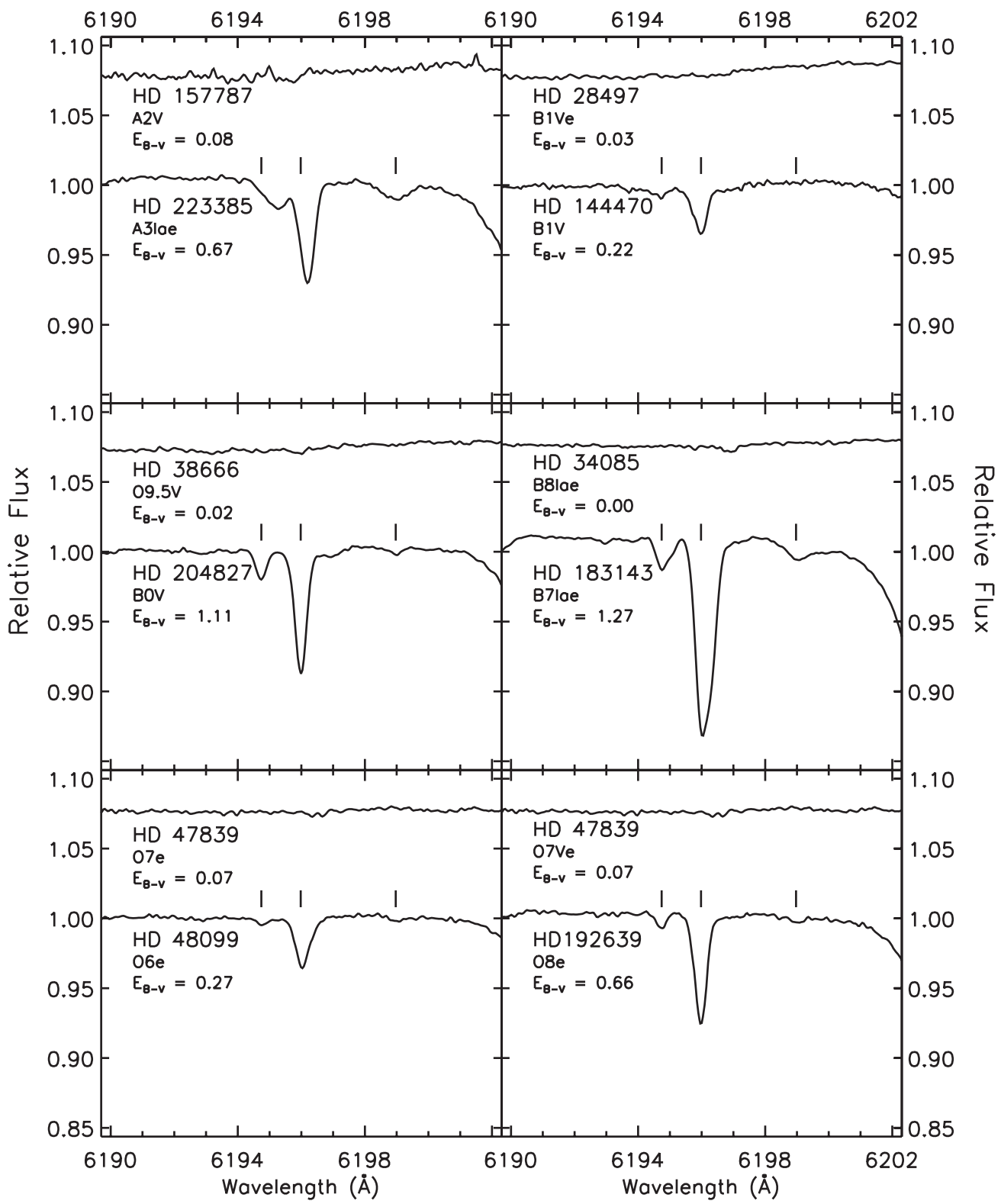

Figure 1. Six examples of the 6196.0 A DIB are shown. The vertical dashes indicate the rest wavelengths from Paper II (Hobbs et al. 2008). Also plotted in each panel are spectra of unreddened stellar comparison stars (offset vertically in flux for clarity) to illustrate the stellar spectrum in the area around each DIB. The drops in the spectra at the longest wavelengths plotted are the wings of a nearby DIB centered at $6204 \AA$. Note that in some cases the DIBs appear to suffer velocity shifts due to the presence of multiple $\mathrm{K}$ I components, as described in the text.

\section{STATISTICAL ANALYSIS}

In this section, we examine the correlation between $\lambda 6196.0$ and $\lambda 6613.6$ in detail. We refer to the measured equivalent width of $\lambda 6613.6$ in the $i$ th sightline (out of $N=114$ sightlines) as $y_{i}$, and that of $\lambda 6196.0$ as $x_{i}$. Our estimated uncertainties for these quantities are denoted $\sigma_{y i}$ and $\sigma_{x i}$, respectively. For a tutorial on linear regression methods, we refer the reader to the review by Feigelson \& Babu (1992), the book by Babu \& Feigelson (1996), and the useful compilation of Press et al. (1997).

\subsection{Ordinary Least Squares Analysis}

By far the most commonly used technique for determining the best-fit line to a set of data is the ordinary least squares algorithm for linear regression. In this approach, it is assumed that there is a relationship $y=\alpha+\beta x$ between the $x$ and $y$ variables, and that (unknown) measurement errors produce deviations from this relationship. The best-fit values of $\alpha$ and $\beta$ are chosen to minimize the quantity $\sum_{i=1}^{N}\left(y_{i}-\alpha-\beta x_{i}\right)^{2}$.

Pearson's correlation coefficient $r$, defined as

$$
r=\frac{\sum_{i=1}^{N}\left(x_{i}-\bar{x}\right)\left(y_{i}-\bar{y}\right)}{\sqrt{\sum_{i=1}^{N}\left(x_{i}-\bar{x}\right)^{2} \sum_{i=1}^{N}\left(y_{i}-\bar{y}\right)^{2}}}
$$

offers a convenient measure of the extent of linear correlation between the two variables. Two variables that are perfectly linearly correlated will have $r=1$, two perfectly anticorrelated variables will have $r=-1$, and two uncorrelated variables will have $r=0$.

Figure 5 shows a plot of $y_{i}$ (the observed equivalent width of $\lambda 6613.6$ ) versus $x_{i}$ (that of $\lambda 6196.0$ ). After applying the ordinary least squares algorithm, we find the best-fit values of 


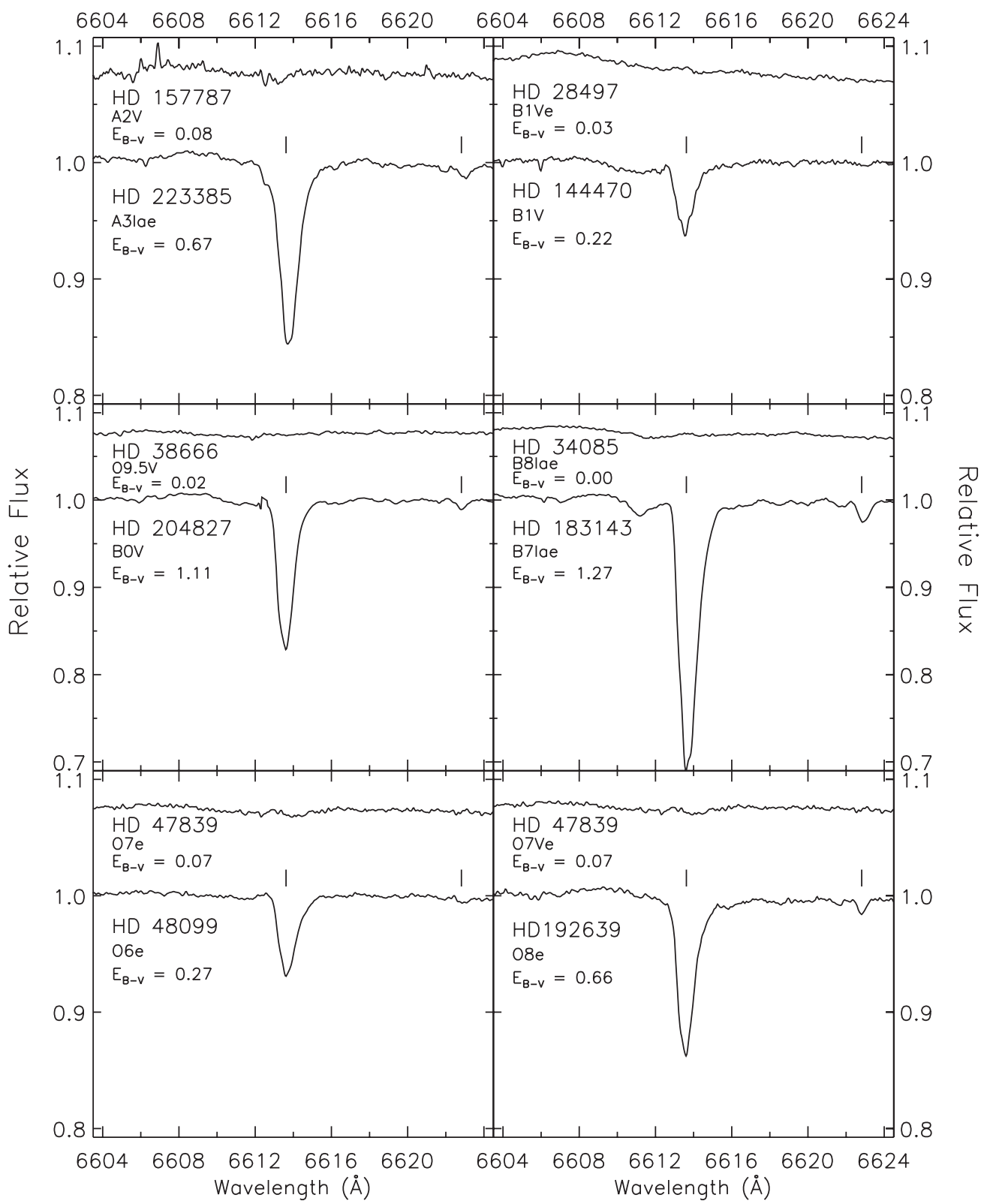

Figure 2. Six examples of the $\lambda 6613.6$ DIB are shown in the same lines of sight, and in the same format, as in Figure 1.

$\alpha=-5.0 \pm 2.2$ and $\beta=3.96 \pm 0.06$, with a correlation coefficient of $r=0.986$. This simple statistical measure indicates a very tight correlation between the equivalent widths of these two DIBs. The value of $r^{2}$ is often taken as a measure of the goodness of fit; in this case $r^{2}=0.971$, indicating that $97.1 \%$ of the variance in the equivalent width of $\lambda 6613.6$ can be explained by the corresponding changes in the equivalent width of $\lambda 6196.0$.

However, the ordinary least squares approach has two major shortcomings. First, it does not treat the two variables symmetrically. If we were instead to regress $x$ on $y$, we would find (taking the relationship $x=\alpha^{\prime}+\beta^{\prime} y$ ) that $\alpha^{\prime}=1.97 \pm 0.52$ and $\beta^{\prime}=0.245 \pm 0.004$; if we invert this relationship to find the values of $\alpha$ and $\beta$ in $y=\alpha+\beta x$, we find $\alpha=-8.0 \pm 2.1$ and $\beta=4.08 \pm 0.07$. Since it is unclear which variable should be regressed on which, this leads to an ambiguity in the fit.

The second major shortcoming of the ordinary least squares approach is that it ignores any knowledge of the uncertainties in the variables. In a case like the present one, we have a priori estimates of the uncertainties (in both $x_{i}$ and $y_{i}$ ) from the continuum fits. Ignoring these uncertainties both prevents appropriate "weighting" of the different observational data points and also leaves unanswered the question of whether the data are statistically consistent with a perfect relationship between the two variables.

\subsection{Maximum Likelihood Functional Relationship Method}

For studying the correlation of two quantities each of which has non-constant but known (or at least estimated) observational errors, the most appropriate approach is one that is variously referred to as a "heteroscedastic functional model" (Feigelson \& Babu 1992), a "heteroscedastic errors-in-variables model" (Babu \& Feigelson 1996), and "maximum likelihood fitting of a functional relationship (MLFR)" (Ripley \& Thompson 1987). The latter reference, derived in the context of testing 


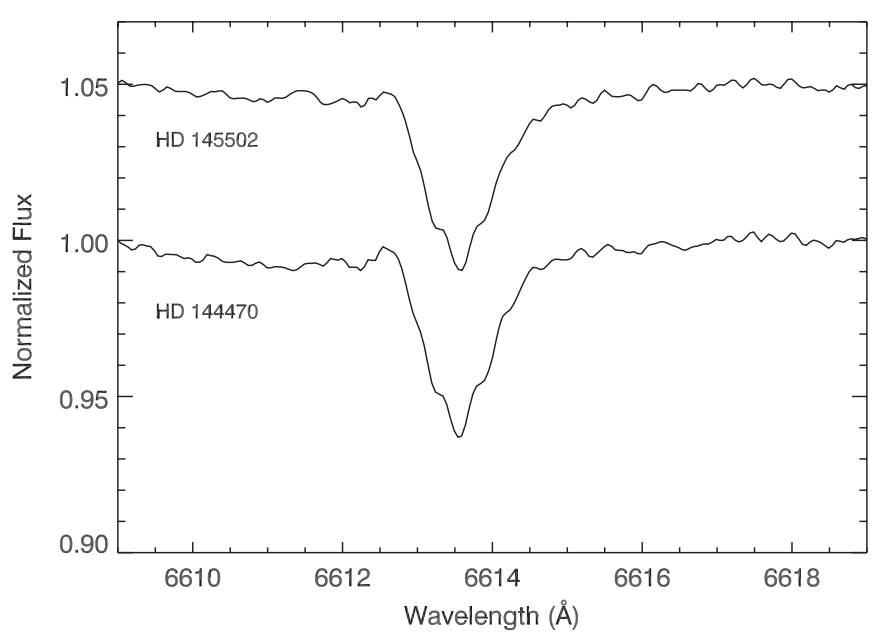

Figure 3. $\lambda 6613.6$ DIB is plotted in two sightlines, HD 144470 and HD 145502. The spectrum for HD 145502 is offset 0.05 vertically in flux. The well-documented substructure can be seen in both of these sightlines.

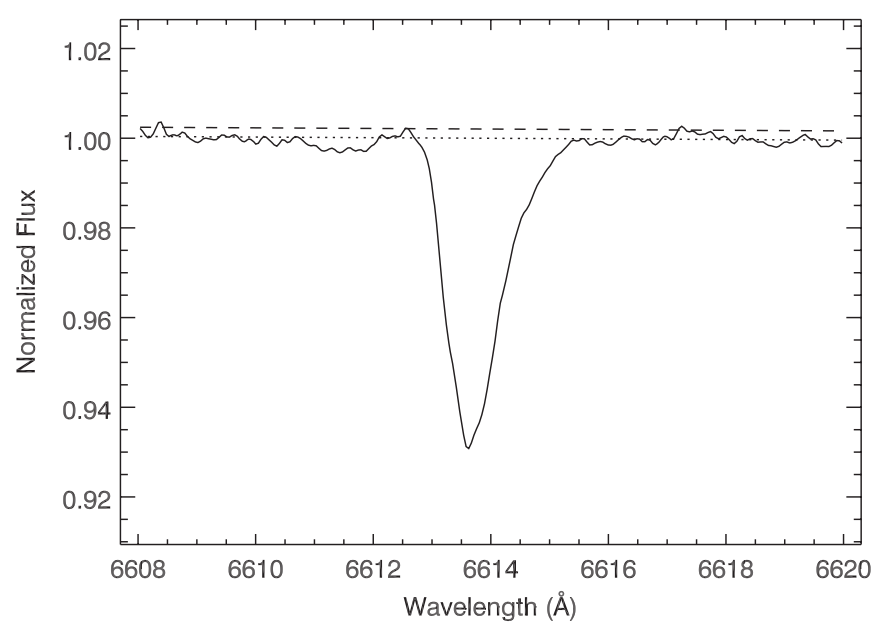

Figure 4. Sample plot of $\lambda 6613.6$ DIB in line of sight toward HD 48099. The two overplotted lines show the best-fit continuum (lower line, dotted) and a continuum displacement of twice our estimated error (upper line, dashed).

and comparing analytical chemistry methods, ${ }^{13}$ contains a very clear derivation and explanation of the method, and we adopt their formalism. This technique was pioneered by York (1966), and has also been implemented in the FITEXY procedure of Press et al. (1997). Because this approach seems not to have been adopted yet by the DIB community, we describe it here in some detail.

In the MLFR method, a true "functional" relationship $v_{i}=$ $\alpha+\beta u_{i}$ is assumed between the actual (unmeasurable) quantities $u_{i}$ and $v_{i}$, rather than between the observed quantities $x_{i}$ and $y_{i}$, which have been contaminated from the true $u_{i}$ and $v_{i}$ by errors that are assumed to be independent and normally distributed with standard deviations $\sigma_{x i}$ and $\sigma_{y i}$. The best estimates $\hat{\alpha}$ and $\hat{\beta}$ are then found by minimizing the quantity

$$
S \equiv \sum_{i=1}^{N}\left[\frac{\left(x_{i}-u_{i}\right)^{2}}{\sigma_{x i}^{2}}+\frac{\left(y_{i}-v_{i}\right)^{2}}{\sigma_{y i}^{2}}\right],
$$

13 This approach has been further explained in a technical brief of the Analytical Methods Committee (No. 10, March 2002) of the Royal Society of Chemistry, which is available along with an Excel add-in at

http://www.rsc.org/Membership/Networking/InterestGroups/Analytical/AMC/ Software/FREML.asp

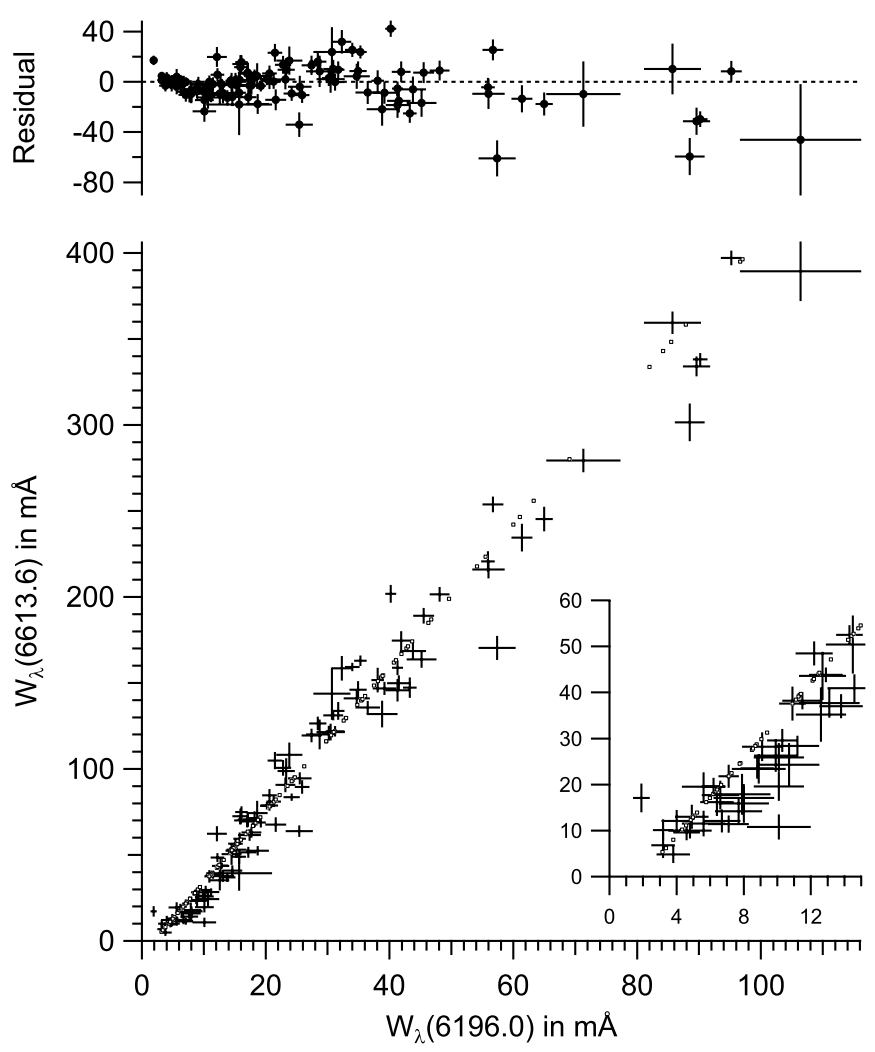

Figure 5. Lower: Correlation $(r=0.986)$ between the equivalent widths of $\lambda 6613.6$ and $\lambda 6196.0$. The size of each cross represents the estimated systematic uncertainty in that measurement. Each square represents the position a data point would have if it were moved onto the best-fit line (which has not been forced to pass through the origin) along the extended diagonal of its error box. Inset: expanded view of the same plot for small values of equivalent width. Upper: plot showing the residuals in the fit of the equivalent width of $\lambda 6613.6 \mathrm{vs}$. that of $\lambda 6196.0$. The sightlines included here cover a wide range of such interstellar parameters as reddening $\left(E_{B-V}=0.02-3.31\right)$ and molecular hydrogen fraction $\left(f_{\mathrm{H}_{2}}^{N}=2.6 \times 10^{-6}-0.76\right)$.

which is derived from the maximum likelihood principle. It can be shown (Ripley \& Thompson 1987) that this quantity is minimized for estimated actual values $\hat{u}_{i}=w_{i}\left[\sigma_{y i}^{2} x_{i}+\sigma_{x i}^{2} \beta\left(y_{i}-\right.\right.$ $\alpha)]$ and $\hat{v}_{i}=\alpha+\beta \hat{u}_{i}$, where $w_{i} \equiv 1 /\left(\sigma_{y i}^{2}+\beta^{2} \sigma_{x i}^{2}\right)$. It then remains to minimize the quantity

$S_{m}(\alpha, \beta)=\sum_{i=1}^{N} S_{i}(\alpha, \beta)^{2}$ where $S_{i}(\alpha, \beta)=\sqrt{w_{i}}\left[y_{i}-\alpha-\beta x_{i}\right]$.

Ripley \& Thompson (1987) further show that $S_{m}$ is minimized for the estimated value of the intercept $\hat{\alpha}=\left[\sum w_{i}\left(y_{i}-\right.\right.$ $\left.\left.\beta x_{i}\right)\right] / \sum w_{i}$; alternatively one may fix $\hat{\alpha}=0$ to force the line through the origin. Given this functional form of $\hat{\alpha}$ as a function of $\beta$, or the assumption that $\hat{\alpha}=0, S_{m}(\beta)$ can then be minimized by numerical methods to find the best estimate of the slope $\hat{\beta}$.

Performing this analysis on our data set, without fixing $\hat{\alpha}=0$, we find $\hat{\alpha}=-7.84 \pm 0.89$ and $\hat{\beta}=4.17 \pm 0.04$. These values agree with the linear regression of $x$ on $y$ described above within their mutual uncertainties, but $\hat{\beta}$ is roughly three combined standard errors ${ }^{14}$ away from the result of the linear regression of $y$ on $x$. For a fit consistent with the assumptions (a perfect

\footnotetext{
${ }^{14}$ The standard errors on the fit parameters, as described in more detail in Press et al. (1997, p. 667), approximately represent the changes in the parameters that result in an increase in $S_{m}$ of unity. Given the large value of $S_{m}$
} in this case, these standard errors should probably not be taken too seriously. 


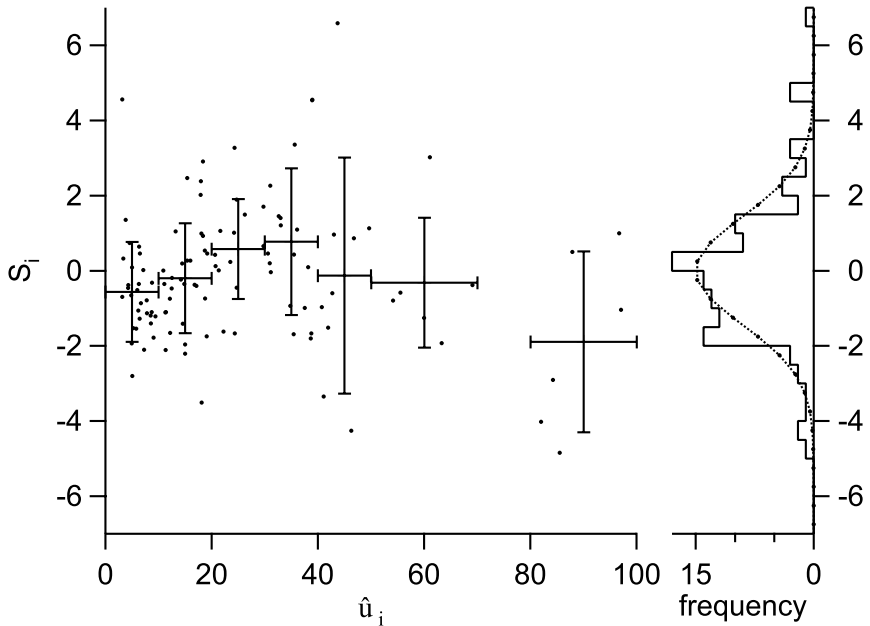

Figure 6. Left: Scaled residuals $S_{i}$ vs. the estimate $\hat{u}_{i}$ of the $\lambda 6196.0$ equivalent width. The crosses with error bars show the average and standard deviation of the $S_{i}$ in a given bin in $\hat{u}_{i}$. No systematic trend, which might indicate a nonlinear relationship, is observed. Right: histogram of the $S_{i}$, along with a Gaussian fit forced to be centered around zero.

functional relationship and correct error estimates), the value of $S_{m} /(N-2)$ should be close to 1 , but we calculate $S_{m}=375$, which leads to $S_{m} / 112=3.35$, considerably larger than 1 .

To quantitatively evaluate the significance of the value of $S_{m}$, we consider the chi-square probability function (often referred to as the $p$-value, but called $Q\left(\chi^{2} \mid v\right)$ in Press et al. 1997 and in Equation (26.4.2) in Abramowitz \& Stegun 1972), which represents the probability that the observed sum-of-squares $S_{m}$ with $v$ degrees of freedom would exceed a certain value based on chance alone if the underlying model (of a perfect correlation contaminated by random errors) were correct. Examining the size of the $p$-value amounts to a test of the null hypothesis that the only source of deviations from a perfect fit is the assumed random errors versus the alternative hypothesis that some other effect is present that increases $S_{m}$ beyond what would be expected from these assumptions. Such effects could include an underestimate of the errors, or a nonlinear relationship between the two quantities being examined. In the present case, the calculated $p$-value is very small, $3.9 \times 10^{-30}$, requiring a rejection of this null hypothesis, and suggesting either that there is not a perfect linear relationship between the two equivalent widths or that our error estimates are incorrect.

A plot of the $S_{i}$ for each sightline versus the best estimate $\hat{u}_{i}$ of the true equivalent width of $\lambda 6196.0$ is shown in Figure 6, along with a histogram of the $S_{i}$. There is no statistically significant systematic trend in these scaled residuals as a function of $\hat{u}_{i}$ to indicate a nonlinear relationship. The residuals appear to be roughly normally distributed, but clearly the distribution of the $S_{i}$ spreads significantly beyond unity.

This analysis suggests that our estimated uncertainties may be too small (or, perhaps, that the assumption of a perfect relationship between these two DIBs is incorrect). Given the difficulties in estimating the uncertainties, it is not unreasonable to expect that we may have underestimated them. If we postulate that the true uncertainties are a factor of 2 higher than our estimates, this decreases $S_{m}$ to $93.8\left(S_{m} /(N-2)=0.84\right.$, now less than unity) and the corresponding $p$-value is 0.89 . In this case, there is an $89 \%$ probability that $S_{m}$ would equal or exceed 93.8 given random errors that are twice our original estimates, and the data are consistent with a perfect linear relationship.
Thus far, we have allowed $\hat{\alpha}$ to be non-zero; if we are to suppose that these two DIBs are due to vibronic transitions in the same molecule, the calculated negative value of $\hat{\alpha}$ would imply that there is another line blended with $\lambda 6196.0$, which is uncorrelated with $\lambda 6613.6$ and $\lambda 6196.0$, and has an average equivalent width of $-\hat{\alpha} / \hat{\beta}=1.9 \mathrm{~m} \AA$. This expression results from inverting the assumed relationship $v=\alpha+u \beta$ to find $u=-\alpha / \beta+v / \beta$. Another possible interpretation could be that there are weak lines interfering (or blended) with both of our target DIBs, and $1.9 \mathrm{~m} \AA$ could represent the average difference between the contamination in the two DIBs. In either case, the interfering line(s) would have to be completely uncorrelated with $\lambda \lambda 6196.0$ and 6613.6.

We have also considered the case of fixing $\hat{\alpha}=0$, that is assuming that there are no interfering lines. In this case, we find $\hat{\beta}=3.94 \pm 0.02$ and $S_{m}=460.4\left(S_{m} /(N-1)=4.07\right)$, which corresponds to a $p$-value of $9.2 \times 10^{-44}$. If we double our error estimates, then $S_{m}=115.1\left(S_{m} /(N-1)=1.02\right)$ or $p=0.40$, which is still a plausible fit. To quantitatively evaluate the significance of the difference between the one-parameter ( $\alpha$ only) and two-parameter $(\alpha, \beta)$ fits, we have performed an "extra sum-of-squares" $F$-test (Montgomery et al. 2006, Equations (3.35) and (8.13)) by calculating the $F$ statistic,

$$
F_{0}=\frac{\left(S_{m}(\alpha)-S_{m}(\alpha, \beta)\right) / S_{m}(\alpha, \beta)}{[(N-1)-(N-2)] /(N-2)]},
$$

which is 25.2. If the more complex two-parameter model did not fit the data significantly better than the simpler one-parameter model, one would expect an $F$ statistic less than unity, so this test confirms the significance of the non-zero value of $\hat{\alpha}$ if the error estimates are correct. However, given the uncertainty in our uncertainties, we do not consider this test as definitively excluding the $\hat{\alpha}=0$ case, since the $p$-value is reasonably high for the case of the doubled uncertainties.

\subsection{Comparison with Other Correlations}

To illustrate how unique the correlation between these two DIBs is, it may be useful to place this relationship in context. Two of the best DIB correlations to date have been shown between (1) the $5780.5 \AA$ DIB and the neutral atomic hydrogen column density $N(\mathrm{H})^{15}$ and (2) numerous DIBs and interstellar $E_{B-V}$ values. For a discussion of the $\lambda 5780.5-N(\mathrm{H})$ correlation, see Herbig (1993); examples of early $E_{B-V}$ correlation discussions can be found in Snow (1973) and Herbig (1975), though numerous individual studies have followed.

Using the same large database of DIB spectra as was used in this work we have equivalent width measurements of the $5780.5 \AA$ DIB (Thorburn et al. 2003, Paper I) and have collected $N(\mathrm{H})$ values from the literature. The subset of stars for which we have both $5780.5 \AA$ DIB equivalent widths and $N(\mathrm{H})$ values is not exactly the same as the group used in the $\lambda 6196.0-\lambda 6613.6$ study, but cover a similarly wide array of interstellar parameters such as interstellar reddening and molecular hydrogen fraction. We use these $5780.5 \AA$ DIB measurements and the corresponding $E_{B-V}$ and $N(\mathrm{H})$ values to evaluate correlation coefficients for these more well established

\footnotetext{
15 In this work, we use $N(\mathrm{H})$ to denote the column density of neutral atomic hydrogen. This should not be confused with $N_{\mathrm{H}}=N\left(\mathrm{H}_{2}\right)+2 N(\mathrm{H})$, which represents the total column density of hydrogen nuclei. The notation $N(\mathrm{H}$ I $)$ is often used instead of $N(\mathrm{H})$, but strictly speaking this usage is incorrect because spectroscopic notation such as $\mathrm{H}$ I should be used only in reference to spectral lines caused by atomic species, not the atomic species themselves.
} 


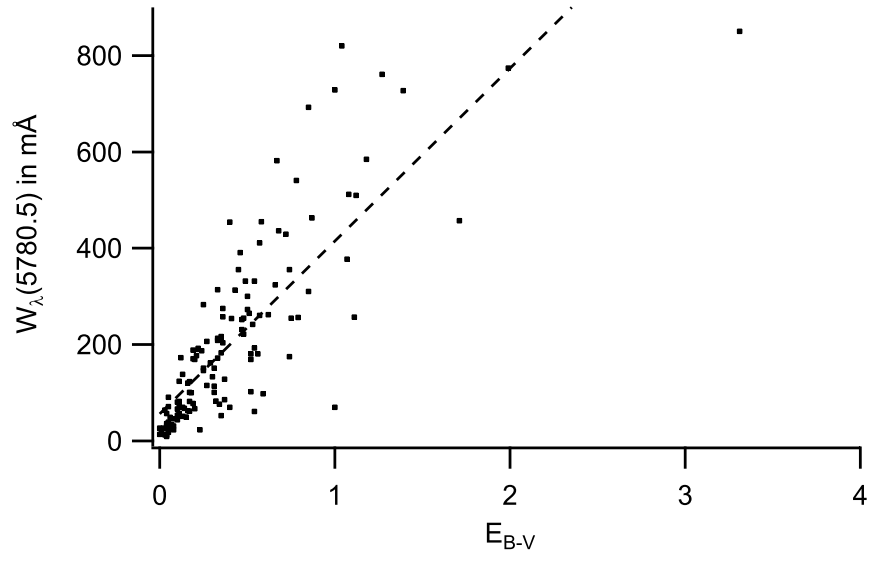

Figure 7. Interstellar $W_{\lambda}(5780.5)$ vs. $E_{B-V}$ values with a correlation coefficient of 0.821 . The dashed line shown is the least-squares fit to the data.

relationships. A more complete study of the correlations among the DIBs will be presented elsewhere (S. D. Friedman et al. 2010, in preparation).

Plots of these two correlations are shown in Figures 7 and 8. The correlation coefficient for the $\lambda 5780.5-E_{B-V}$ relationship is $r=0.821$. This is a good relationship, though it is not nearly good enough that the $E_{B-V}$ could be used as a strict predictor of the $5780.5 \AA \mathrm{DIB}$ strength. Given an $r$ value of 0.821 , only $67.4 \%$ of the variance in the $\lambda 5780.5$ equivalent width measurements can be accounted for by changes in $E_{B-V}$.

For the $\lambda 5780.5-N(\mathrm{H})$ correlation, we plot both the equivalent width of $\lambda 5780.5$ and the neutral atomic hydrogen column density in log space, and exclude from the analysis the three sightlines labeled in Figure 8 which are obvious outliers (and were also discrepant in the analysis of Herbig 1993). The resulting correlation coefficient for this pair is 0.953 , considerably better than the $\lambda 5780.5-E_{B-V}$ association and quite a good relationship in its own right. Yet, even after plotting on a log-log plot and rejecting three outliers, this correlation still is not as strong as that found for the $\lambda 6613.6$ and $\lambda 6196.0$ DIBs. When the outliers are included, obviously the correlation worsens still, yielding a correlation coefficient of 0.901 .

We have also examined the correlation in our spectral database between the equivalent width of the $A-X 1-0$ and $0-0 R(0)$ transitions of $\mathrm{CH}^{+}$at 3957.5 and $4232.5 \AA$, respectively (see Figure 9). These two individual rovibronic transitions of $\mathrm{CH}^{+}$arise from the same rotational level in the ground state, and consequently they should exhibit a perfect correlation in the absence of observational errors. Including all 56 sightlines in which we detected both transitions, the correlation coefficient is 0.985 ; the corresponding $p$-value is 0.02 . If the two sightlines with highest equivalent widths (most likely to suffer saturation) are excluded, the correlation coefficient remains quite similar (0.983) but the $p$-value increases to 0.12 . These $p$-values are considerably higher than those of the $\lambda 6196.0 / 6613.6$ pair, which may be partly due to the fact that these lines are narrow and isolated whereas the DIBs are more subject to interfering transitions and continuum placement uncertainties. Even so, the $p$-values for the pair of $\mathrm{CH}^{+}$transitions are still significantly lower than unity, which is further evidence that we may have underestimated our measurement uncertainties.

\section{CONCLUSIONS}

There is no question that the two DIBs $\lambda 6196.0$ and $\lambda 6613.6$ are very well correlated. Their correlation is better than any other

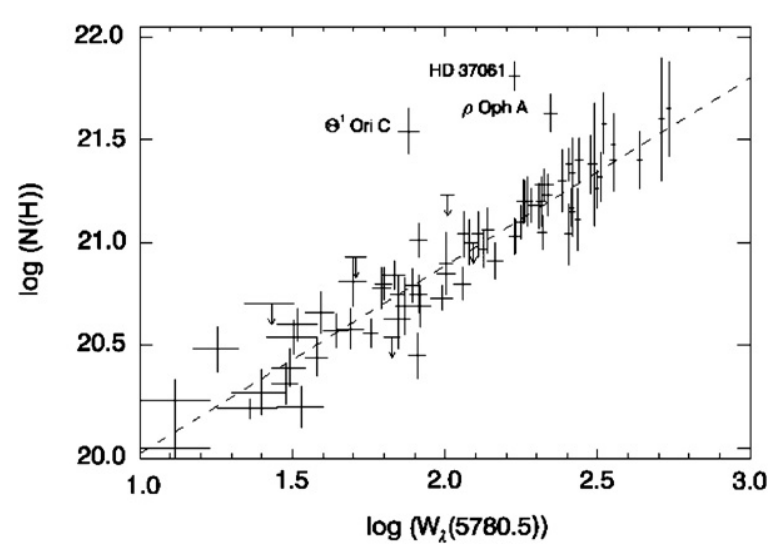

Figure 8. $\log -\log$ plot of column density of atomic hydrogen vs. $W_{\lambda}(5780.5)$ The correlation is generally considered to be good, with a correlation coefficient of 0.953 when the three labeled outliers are excluded. The least-squares fit line is plotted as the dashed line in the figure; this fit is not forced to pass through the origin.

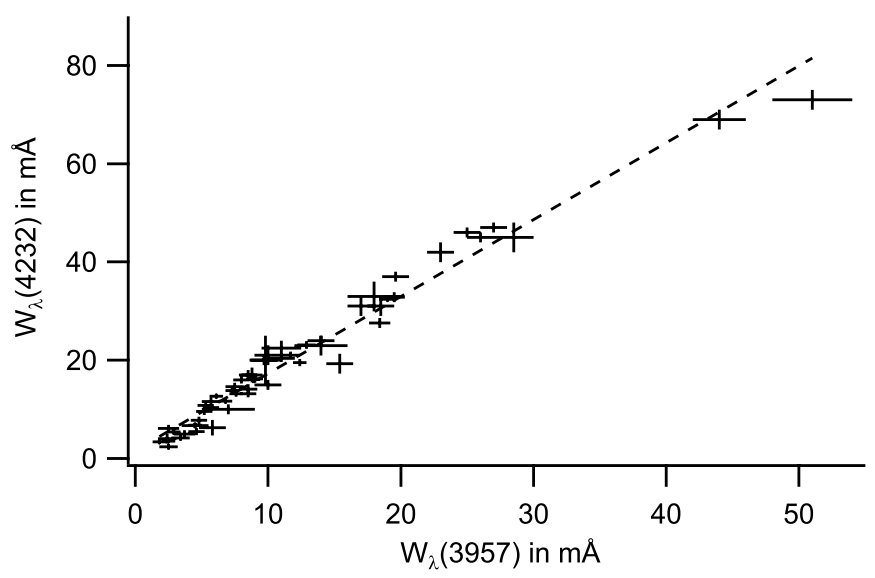

Figure 9. Correlation between the equivalent widths of the two $R(0)$ transitions of $\mathrm{CH}^{+}$at 3957.5 and $4232.5 \AA$, which should be perfectly correlated because they arise from the same rotational level in the ground state. The observed correlation coefficient is 0.985 .

correlation among DIBs and is, to the best of our knowledge, the best correlation ever observed for interstellar quantities. The critical question that remains is whether these two DIBs are due to the same molecular carrier.

If the two DIBs are vibronic transitions of the same molecule, then the observed deviations from a perfect correlation must be due to errors in the measurements. The largest source of these errors appears to be systematic errors such as uncertain continuum placement and contributions from blended lines. Such blended lines could potentially be weak, as yet undetected, DIBs caused by other molecular carriers. We have adopted a very crude error estimate based on continuum placement errors, and the statistical analysis in Section 4.2 shows that these two DIBs could be perfectly correlated if we have underestimated our errors by only a factor of 2 . Such an underestimation strikes us as entirely plausible, considering how difficult these error sources are to quantify.

If the two DIBs $\lambda 6196.0$ and $\lambda 6613.6$ are indeed caused by the same electronic transition of the same molecule, the ratio of their Franck-Condon factors is $\sim 1: 4$. The difference between the vibronic band positions of $6195.98 \AA$ and $6613.62 \AA$ would correspond to a vibrational frequency in the electronically excited state of $1018.9 \mathrm{~cm}^{-1}$, a value consistent with that reported by Moutou et al. (1999). It will be very interesting to search for other DIBs that are tightly correlated with $\lambda 6196.0$ 
and $\lambda 6613.6$ and might represent additional vibronic transitions of their common carrier.

Despite the nearly perfect correlation between these two DIBs, there is a potential spectroscopic problem with concluding that they share a common molecular carrier: they do not have the same shape and width. In fact, the two DIBs have linewidths that are quite different (by a factor of $\sim 2$ ) when observed with high resolution (Galazutdinov et al. 2002), and also have rather different lineshapes. It is well known (e.g., Ueda \& Shimanouchi 1968) that different vibronic (or vibrational) transitions of a given asymmetric top molecule can exhibit different band shapes for bands whose transition dipoles lie along different axes (socalled A-type, B-type, and C-type transitions). In the simulations of Ueda \& Shimanouchi (1968), it is also evident that the overall widths of those bands can vary to some extent. Thus, it seems conceivable that these two DIBs could be due to the same molecule, but a set of molecular constants that can reproduce both band profiles (in theoretical spectral simulations) will need to be identified before it can be established that the two DIBs do share the same carrier.

If these two DIBs are not transitions of the same molecular carrier, then the close correlation between these two DIBs does imply at the very least that the two carriers themselves must be well correlated. In principle, two different molecules whose abundances maintain exactly the same ratio regardless of the local physical and chemical conditions would also produce a perfect correlation. However, the two molecules would need to be so closely related that their chemistry is nearly identical in order for the abundance ratios to remain the same across such a wide range of environments. To our knowledge, such a tight correlation between two interstellar molecules has never been observed before, and would represent a remarkable result on its own even apart from the implications for remaining DIB carriers.

The relationship of these two DIBs remains an enigma. If they are due to the same carrier, how can we understand their very different profiles? If they are due to two different molecular carriers, what sort of chemical pathways can ensure that their relative abundance is nearly identical over such a wide range of interstellar conditions? Further insight on this problem may come from four different lines of inquiry. First, a more thorough investigation of potential error sources in the current survey may provide better estimates of the uncertainties. Second, a search for some sightline parameter that correlates with the residuals observed in the correlation may provide clues about the existence of interfering lines. Third, future astronomical measurements of these two DIBs with higher $\mathrm{S} / \mathrm{Ns}$, and preferably with higher resolving power to help resolve interfering lines, in a similarly large sample of sightlines, could offer a definitive answer to whether the correlation of these two DIBs is perfect. Fourth, a theoretical explanation of how two vibronic bands could (or could not) produce such different profiles would provide a plausibility argument for (or compelling evidence against) the conclusion that these two DIBs share a common carrier.

We gratefully acknowledge the suggestions by E. C. Sutton regarding the statistical methods for studying the DIB correlations, as well as helpful discussions with T. Oka regarding the spectroscopic objections to these two DIBs sharing a common carrier. A portion of this work was performed while M.M.D. was visiting the University of Illinois, while supported by a NASA
Graduate Student Researchers Program grant (NGT2-52300). B.J.M. gratefully acknowledges support from the David and Lucile Packard Foundation and the University of Illinois.

Facilities: Apache Point Observatory.

\section{REFERENCES}

Abramowitz, M., \& Stegun, I. A. 1972, Handbook of Mathematical Functions (New York: Dover)

Babu, G. J., \& Feigelson, E. D. 1996, Astrostatistics (London: Chapman \& Hall) Bohlin, R. C., Savage, B. D., \& Drake, J. F. 1978, ApJ, 224, 132

Cami, J., Salama, F., Jiménez-Vicente, J., Galazutdinov, G. A., \& Krełowski, J. 2004, ApJ, 611, L113

Cami, J., Sonnentrucker, P., Ehrenfreund, P., \& Foing, B.H. 1997, A\&A, 326, 822

Diplas, A., \& Savage, B. D. 1994, ApJS, 93, 211

Ehrenfreund, P., \& Foing, B. H. 1996, A\&A, 307, L25

Feigelson, E. D., \& Babu, G. J. 1992, ApJ, 397, 55

Galazutdinov, G. A., LoCurto, G., Han, I., \& Krełowski, J. 2008a, PASP, 120, 178

Galazutdinov, G. A., LoCurto, G., \& Krełowski, J. 2008b, ApJ, 682, 1076

Galazutdinov, G. A., LoCurto, G., \& Krełowski, J. 2008c, MNRAS, 386, 2003

Galazutdinov, G., Manico, G., Pirronello, V., \& Krełowski, J. 2004, MNRAS, 355,169

Galazutdinov, G., Moutou, C., Musaev, F., \& Krełowski, J. 2002, A\&A, 384, 215

Heger, M. L. 1922, Lick Obs. Bull., 337, 146

Herbig, G. H. 1975, ApJ, 196, 129

Herbig, G. H. 1993, ApJ, 407, 142

Herbig, G. H. 1995, ARA\&A, 33, 19

Herbig, G. H., \& Soderblom, D. R. 1982, ApJ, 252, 610

Herzberg, G. H. 1950, Molecular Spectra and Molecular Structure, Vol. I., Spectra of Diatomic Molecules (New York: Van Nostrand Reinhold)

Hobbs, L. M., et al. 2008, ApJ, 680, 1256 (Paper II)

Hobbs, L. M., et al. 2009, ApJ, 705, 32 (Paper III)

Jenniskens, P., \& Désert, F.-X. 1994, A\&AS, 106, 39

Johnson, H. L. 1963, in Basic Astronomical Data, ed. K. A. Strand (Chicago, IL: Univ. Chicago Press), 214

Josafatsson, K., \& Snow, T.P. 1987, ApJ, 319, 436

Kerr, T. H., Hibbins, R. E., Miles, J. R., Fossey, S. J., Somerville, W. B., \& Sarre, P. J. 1996, MNRAS, 283, L105

Merrill, P. W. 1934, PASP, 46, 206

Merrill, P. W., \& Wilson, O. C. 1938, ApJ, 87, 9

Montgomery, D. C., Peck, E. A., \& Vining, G. G. 2006, Introduction to Linear Regression Analysis (New York: Wiley Interscience)

Moutou, C., Krełowski, J., d'Hendecourt, L., \& Jamroszczak, J. 1999, A\&A, 351,680

Press, W. H., Teukolsky, S. A., Vetterling, W. T., \& Flannery, B. P. 1997, Numerical Recipes in C (Cambridge: Cambridge Univ. Press)

Rachford, B. L., et al. 2002, ApJ, 577, 221

Rachford, B. L., et al. 2009, ApJS, 180, 125

Ripley, B. D., \& Thompson, M. 1987, Analyst, 112, 377

Sarre, P. J. 2006, J. Mol. Spectrosc., 238, 1

Sarre, P. J., Miles, J. R., Kerr, T. H., Hibbins, R. E., Fossey, S. J., \& Somerville, W. B. 1995, MNRAS, 277, 41

Savage, B. D., Bohlin, R. C., Drake, J. F., \& Budich, W. 1977, ApJ, 216, 291

Sembach, K. R., \& Savage, B. D. 1992, ApJ, 83, 147

Shull, J. M., \& van Steenberg, M. E. 1985, ApJ, 294, 599

Smith, W. H., Snow, T. P., Jura, M., \& Cochran, W. D. 1981, ApJ, 248, 128

Smith, W. H., Snow, T. P., \& York, D. G. 1977, ApJ, 218, 124

Snow, T. P. 1973, AJ, 78, 913

Snow, T. P., \& McCall, B. J. 2006, ARA\&A, 44, 367

Sonnentrucker, P., Foing, B. H., Breitfellner, M., \& Ehrenfruend, P. 1999, A\&A, 346, 936

Spitzer, L., Cochran, W. D., \& Hirshfeld, A. 1974, ApJS, 28, 373

Thorburn, J. A., et al. 2003, ApJ, 584, 339 (Paper I)

Ueda, T., \& Shimanouchi, T. 1968, J. Mol. Spectrosc., 28, 350

Walker, G. A. H., Bohlender, D. A., \& Krełowski, J. 2000, ApJ, 530, 362

Wang, S., et al. 2003, Proc. SPIE, 4841, 1145

Webster, A. 1996, MNRAS, 282, 1372

Welter, G. L., \& Savage, B. D. 1977, ApJ, 215, 788

Weselak, T., Galazutdinov, G. A., Musaev, F. A., \& Krełowski, J. 2008, A\&A, 484, 381

York, D. 1966, Can. J. Phys., 44, 1079 